\title{
Investigation of Possible Nucleation Mechanisms for Producing an Ultra-Refined Alpha Phase Microstructure in Beta Titanium Alloys Using High-Resolution Electron Microscopy and 3D Atom Probe Tomography
}

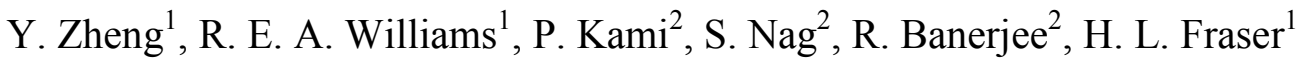 \\ 1. Department of Material Science and Engineering, The Ohio State University, 43026 \\ 2. Department of Materials Science and Engineering, University of North Texas, 76203
}

Titanium alloys contain complex, interdependent microstructural features that can range from $\mathrm{nm}$ to $\mathrm{mm}$ in length. In order to fully probe the salient microstructural features across this variation in length scale, multiple characterization techniques must be implemented and correlated. For the work to be presented, conventional transmission electron microscopy (CTEM), selected area diffraction (SAD), scanning transmission electron microscopy (STEM) utilizing Z-contrast imaging and apparent atomic resolution EDS mapping as well as 3D atom probe tomography (3DAP) were all correlated to fully characterize the micro- and nano- meter structural features, as well as compositional variations, that might possibly influence alpha phase nucleation. Moreover, the efforts of this research lead to the discovery of a previously unknown phase that is believed to play an impactful role in alpha phase nucleation.

Due to its high strength and deep-hardenability, Ti-5Al-5Mo-5V-3Cr (Ti5553) is an excellent metastable $\beta$ titanium alloy with significant usage in landing gear components. The strength, ductility and fracture toughness of Ti5553 can be modified over a wide range by varying thermo- mechanical history to control the size scale and distribution of the $\alpha$ phase (hcp) from the $\beta$ matrix (bcc) due to the critical role it plays in determining material properties. One important factor that may influence $\alpha$ precipitation is compositional instability and/or structural instability within the $\beta$ matrix, such as instabilities formed due to the presence of metastable phases within the $\beta$ matrix[1,2]. $\omega$ phase, which exhibits hexagonal structure and rejects solute into the $\beta$ matrix, can be treated as both a compositional and structural instability within the $\beta$ matrix that might influence the driving force of $\alpha$ precipitation by imposing an extra compositional and/or stress field[3-5]. Therefore, it is critical to obtain a detailed understanding of the metastable phases present prior to stable $\alpha$ phase precipitation in order to manipulate Ti5333 for specific engineering applications and help understand alpha nucleation mechanisms.

Figure 1 shows 3DAP, dark-field TEM, and HAADF-STEM imaging of the isothermal $\omega$ phase indicating that coarsening arrests when $\omega$ particles are approximately $10 \mathrm{~nm}$ along the long axis and $5 \mathrm{~nm}$ in short axis direction; HAADF-STEM imaging confirms $\{111\}$ atom planes collapse is complete within the core of isothermal $\omega$ particles as well as interfacial coherency with the matrix. Additionally, 3DAP and STEM-EDX reveal that all solute was rejected into $\beta$ matrix during isothermal $\omega$ growth. Figure 3 shows a HAADF-STEM image of super-refined $\alpha$ apparently nucleating at the $\omega / \beta$ interface. Interfacial ledges were observed by HAADF-STEM at $\omega / \beta$ interface and could act as favorable nucleation sites for super-refined $\alpha$. Supplemental data also shows $\alpha$ nucleation in the $\beta$ phase regions near $\omega$ particles where local composition varies. 
During characterization of Ti5553, a new phase of ordered, face-centered orthorhombic structure was found using CTEM, SAD, and HRSTEM-HAADF images as shown in Figure 4. The lattice parameters of this unknown phase are determined to be $\mathrm{a}=0.328 \mathrm{~nm}, \mathrm{~b}=0.464 \mathrm{~nm}$ and $\mathrm{c}=1.393 \mathrm{~nm}, \alpha$ $=\beta=\gamma=90^{\circ}$, space group Fmmm. The orientation relationship with the $\beta$ phase was measured: $(001)_{\text {ordered orthorhombic }} / /(011)_{\mathrm{BCC}}$ and $[100]_{\text {ordered orthorhombic }} / /[100]_{\mathrm{BCC}}$. Figure 4 shows the negligible misfit between ordered face centered orthorhombic phase and $\beta$ phase forming essentially coherent interfaces.

\section{References:}

\section{[1] Y. Zheng, et al, Unpublished Research}

[2] Nag, S., et al, Acta Materialia, 60 (2012) p. 6247-6256.

[3] Devaraj, A., et al, Acta Materialia, 60 (2012). p. 596-609.

[4] Devaraj, A., et al, Scripta Materialia, 61 (2009): p. 701-704.

[5] Y. Zheng, et al, Unpublished Research.

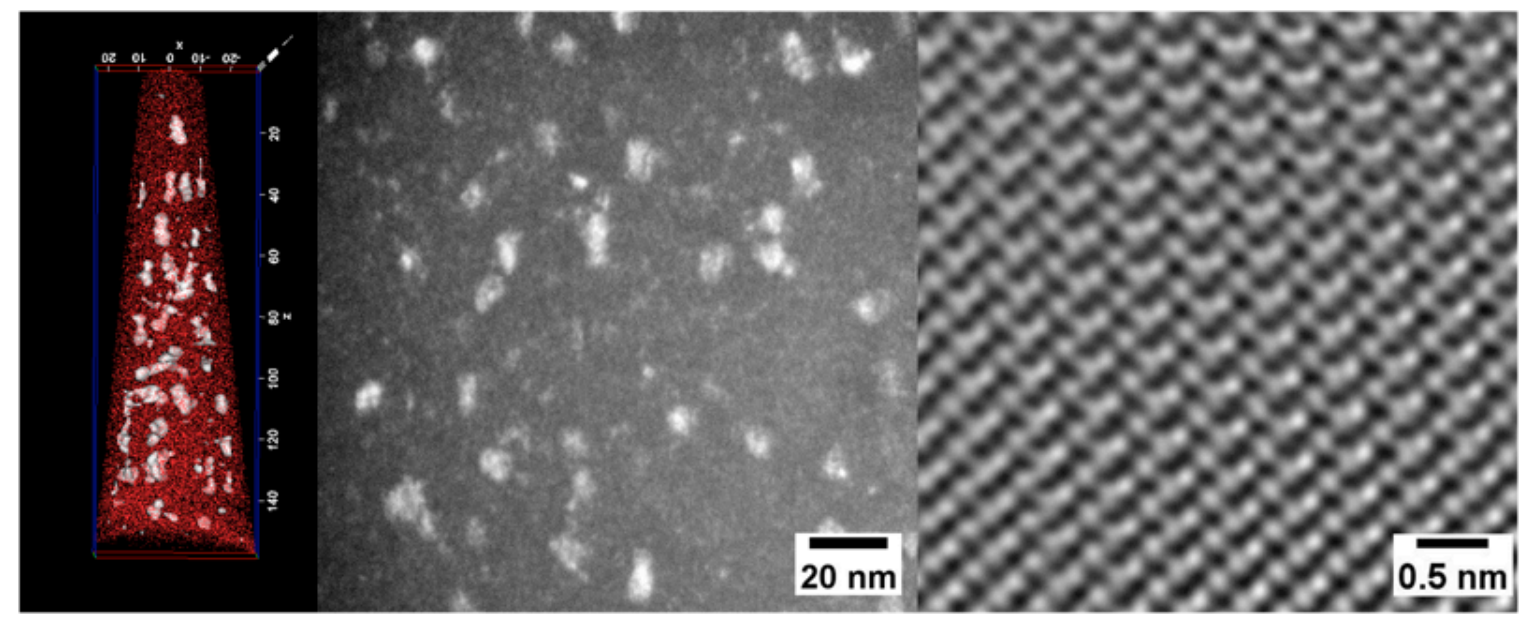

Figure 1: Characterization of isothermal $\omega$ by (a) 3DAP, (b) dark-field TEM, (c) HAADF-STEM

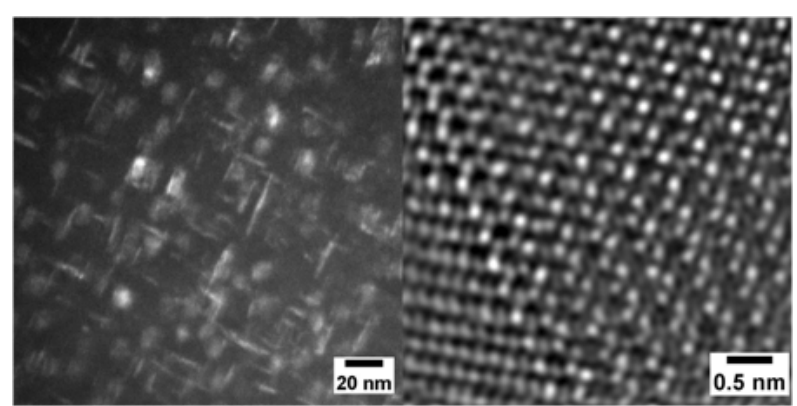

Figure 2: (a) Dark-field TEM image showing the coexistence of $\alpha, \omega$, and $\beta$, (b) HAADF-STEM image showing the apparent intersection of $\alpha / \omega$

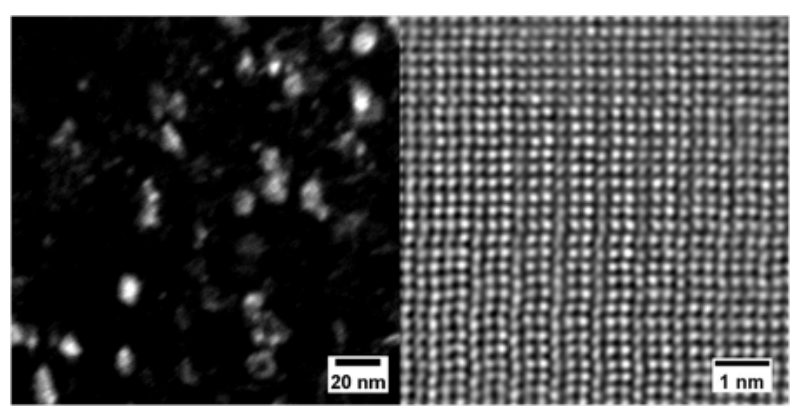

Figure 3: Imaging of unknown phase using (a) dark-field TEM and (b) HAADF-STEM to reveal morphology and structure 